\title{
Finite-Time Thermodynamics and the Optimal Control of Chemical Syntheses
}

\begin{tabular}{|r|l|}
\hline Journal: & Zeitschrift für Anorganische und Allgemeine Chemie \\
\hline Manuscript ID: & zaac. 200900207.R1 \\
\hline Wiley - Manuscript type: & Article \\
\hline Date Submitted by the \\
Author: & 02-Jun-2009 \\
\hline Complete List of Authors: & $\begin{array}{l}\text { Schoen, Christian; MPI fuer Festkoerperforschung Stuttgart, } \\
\text { Abteilung Jansen, Chemie III }\end{array}$ \\
\hline Keywords: & $\begin{array}{l}\text { Finite-time thermodynamics, optimal control, nucleation-and- } \\
\text { growth, chemical syntheses }\end{array}$ \\
\hline
\end{tabular}

\section{S scholaroNE \\ Manuscript Central}


1

2

3

4

5

7

8

9

10

11

12

13

Finite-Time Thermodynamics and the Optimal Control of Chemical Syntheses

\author{
J. Christian Schön*
}

Max-Planck-Institut für Festkörperforschung, D-70569 Stuttgart

Dedicated to Profesor Dr. Dr. h.c. Martin Jansen on his 65th birthday

*Prof. Dr. J. Christian Schön

Max-Planck-Institut für Festkörperforschung

Heisenbergstr. 1

D-70569 Stuttgart

e-mail: schoen@fkf.mpg.de

Keywords: Finite-time thermodynamics, optimal control, nucleation-and-growth, chemical syntheses 


\begin{abstract}
:
The optimization of chemical processes that take place in a finite time constitutes an important application of finite-time thermodynamics. In this study we investigate two generic optimal control problems for nucleation-and-growth based syntheses: the maximization of the amount of a crystalline solid phase generated via cooling from the melt within a finite time $\tau$, and the maximization of the difference between two metastable crystalline modifications again synthesized by crystallization from a supercooled melt. In both cases the optimal temperature program consists in a bangbang solution with constant values of the temperature, where a switch from a temperature $T_{1}$, where nucleation rates are high, to a temperature $T_{0}>T_{1}$, where the growth rates of the crystallites are maximal, occurs. The location of the switching time $t_{s}^{*}, 0 \leq t_{s}^{*} \leq \tau$, is analyzed as function of the parameters of the models describing the chemical systems, and an application to the synthesis of glycerol crystals is given.
\end{abstract}

Inhaltsübersicht:

Die Optimierung chemischer Prozesse, die in endlicher Zeit ablaufen, stellt eine wichtige Anwendung der „Thermodynamik in endlicher Zeit“ dar. In dieser Arbeit untersuchen wir zwei generische Probleme der optimalen Kontrolle für Synthesen, die auf Keimbildung und -wachstum basieren: Die Maximierung der Menge einer kristallinen festen Phase, die durch Abkühlung einer Schmelze in einer endlichen Zeit $\tau$ entsteht, sowie die Maximierung der Differenz zweier metastabiler Modifikationen, die ebenfalls durch Kristallisierung aus einer unterkühlten Schmelze gewonnen werden. In beiden Fällen ergibt sich für das optimale Temperaturprogramm eine Bang-Bang- 
Lösung mit konstanten Temperaturwerten, bei der ein Sprung von einer Temperatur $T_{1}$, bei der die Keimbildungsraten hoch sind, zu einer Temperatur $T_{0}>T_{1}$, bei der die Wachstumsraten der Keime maximal sind, auftritt. Der Zeitpunkt des Sprungs $t_{s}^{*}$, $0 \leq t_{s}^{*} \leq \tau$, wird als Funktion der Modellparameter der chemischen Systeme analysiert, und eine Anwendung auf die Synthese von Glycerolkristallen wird gegeben. 
1. Introduction

The major task of experimental chemistry has been, and still is, the synthesis of new compounds, molecules and solids, closely followed by the determination of the mechanisms that underlie the individual reactions and the whole synthesis process. Optimizing these syntheses with regard to an increased yield or a decreased work consumption is usually a secondary issue to the ability to synthesize the compound in the first place.

In contrast, the optimization of chemical processes constitutes one of the major tasks in the field of chemical engineering [1]. Besides the straightforward task of optimizing individual chemical reactions [2-6] or a sequence of reactions [7], the most common examples are the increase in the efficiency of various distillation procedures [8-10], the design of chemical plants [11], where complex syntheses take place that include e.g. heat exchanger networks [12] or the recycling of chemicals, and the transformation between different phases of a given substance with a minimal loss of availability [13]. Typically, the objective(s) or cost function(s) with respect to which a chemical process is to be optimized are the yield of the desired product, the amount of chemicals needed and the energy required for the production, the total monetary cost, or the environmental impact of the process.

In many, perhaps most, instances, such an optimization is based on empirical rules of thumb, where one is often guided by simple estimates and models that capture (at least qualitatively) some basic features of the reactions involved. Similarly, one often proceeds by systematically varying the process parameters in the laboratory where 
efficient combinatorial schemes and correlation tables are employed to reduce the amount of test syntheses needed [14] and to control multivariate processes [15]. An example of such systematic variations of process parameters are the so-called highthroughput syntheses $[16,17]$. They are most efficient, if one wants to optimize some property within a well-defined class of molecular or solid compounds; in this way they nicely complement the theoretical scanning of the energy landscapes of chemical systems, where the primary goal is to discover the possible types of compounds that can serve as synthesis targets in a given chemical system [18-21].

\begin{abstract}
Alternatively, one can address this issue on the level of theory. The general mathematical theory that underlies such an optimization is the so-called optimal control theory [22]. Here, one describes the system by a set of ,internal“" variables $\vec{x}(t)$ plus a set of controls $\vec{u}(t)$ that can be adjusted within certain limits to achieve an optimal outcome of the process. This optimal control problem is then usually solved using the calculus of variations or dynamical programming methods.
\end{abstract}

One notes that for many chemical processes the objective function is a thermodynamic quantity such as the total amount of a substance, the entropy produced or the work consumed in the process. On a very general level, the optimal control of such processes falls into the purview of the field of finite-time thermodynamics (FTT) [23]. Finite-time thermodynamics deals with the implications for thermodynamic processes of having only a finite time available to achieve a certain task, e.g. performing a complete cycle of a thermodynamic engine such as the Carnot- [24] or the Otto-engine [25]. In particular, a FTT-analysis yields both a lower (or upper) bound on the thermodynamic quantity of 
interest and the optimal path (in control variable space, and thus in internal variable space) that achieves this limit value.

In this paper, we will give an introduction to the field of finite-time thermodynamics and optimal control, and show some applications in the field of chemistry. In particular, we will discuss the application of optimal control to the optimization of the outcome of a generic synthesis that proceeds via nucleation-and-growth processes, where one or two (metastable) compounds can be the outcome of the synthesis.

\section{Finite-Time Thermodynamics}

Classical thermodynamics deals with the analysis of thermodynamic processes, which are defined as the movement of a chemical or physical system between thermodynamic equilibrium states. Using the first and second law of thermodynamics and the concept of reversible and irreversible quasi-static processes, it is possible to define state functions in the thermodynamic space and to derive bounds on thermodynamic processes, in particular on cyclic processes. However, one of the basic assumptions behind this analysis is that an infinite time is available for the processes to take place, together with the ability to perform the process in infinitesimally small increments along the path.

The driving force behind the development of the so-called finite-time thermodynamics was the recognition that these bounds on the efficiency or maximum power of an ideal cyclic process are of only limited relevance for real processes where only a finite time (and / or a finite number of steps) is available for completing the cycle. Until the middle of the seventies of the last century, the focus was on the so-called second-law analysis 
$[26,27]$ that was employed predominantly in the engineering sciences, in order to develop (economically) efficient procedures. This changed with the work by Curzon \& Ahlborn [28], who considered the (global) optimization of thermodynamic processes in a finite time interval.

In nearly all application-oriented questions in finite-time thermodynamics, one deals with an optimal control problem [22], where a quantity $J[\vec{x}(t), \vec{u}(t), t]$ is to be optimized that is given as a functional of the internal (thermodynamic) variables of the system $\vec{x}(t)$ and the control parameters $\vec{u}(t)$. The time evolution of these variables is usually described by differential equations

$d x / d t=\stackrel{r}{f}(\stackrel{r}{x}, \stackrel{r}{u}, t)(1)$

that often represent phenomenological rates of change or dissipation equations. In addition, the process has to obey certain boundary conditions, which often take the form $\dot{x}\left(t_{f}\right)=\dot{x}_{f}, \dot{x}\left(t_{0}\right)=\dot{x}_{0} ; t_{0}=0, t_{f}=\tau$.

A short introduction into the concepts of optimal control theory is given in the supplementary material. By now a large number of thermodynamic processes have been analysed, e.g. Carnot-cycles [24,29,30], Diesel-cycles [31], Otto-cycles [25], heat exchange [12], diffusion [32], energy conversion [30], phase conversion [33], distillation [8-10], heat pumps and refrigerators [34-36], thermal insulation [37], solar energy [38], chemical reactions [6,7] and chemical converters [1]. Over the past two decades, this analysis has been performed for engines that use not only classical gases as 
medium but also Bose- and Fermi-gases [39,40], and has been extended to a large variety of multi-source systems and complicated dissipative systems [41]. Furthermore, spatially dispersed systems have been investigated, where both the thermodynamic variables and the control parameters can vary in space [42], and the performance of computer algorithms has been optimized [43]. ${ }^{1}$

One reason for the broad applicability of finite-time thermodynamics are some very general and generalizable concepts that were introduced in the early eighties. These are based on the insight that one can compute very general bounds on the quantities to be minimized [47], e.g. the increase of entropy $\Delta S^{u}$ or the loss of availability $-\Delta A^{u}$,

$$
\Delta S^{u} \geq \frac{\bar{r}}{\tau} L_{S}^{2} \text { and }-\Delta A^{u} \geq \frac{\bar{r}}{\tau} L_{U}^{2}
$$

which depend only on the thermodynamic path length

$$
d L_{S}(d \vec{X})=\sqrt{\sum_{i, j} d X_{i} \frac{\partial^{2} S(\vec{X})}{\partial X_{i} \partial X_{j}} d X_{j}} \text { and } d L_{U}(d \vec{X})=\sqrt{\sum_{i, j} d X_{i} \frac{\partial^{2} U(\vec{X})}{\partial X_{i} \partial X_{j}} d X_{j}}
$$

between the initial and final point in thermodynamic space defined via the matrix of second partial derivatives of $U(S, V, \ldots)$ and $S(U, V, \ldots)$, respectively, $[48,49]$ and the various parameters in the phenomenological evolution equations such as the relaxation time $\bar{r}$ and the total time $\tau$ of the process. For more details we refer the reader to the supplementary material.

\footnotetext{
${ }^{1}$ Even outside the fields of physics, chemistry and engineering, a variety of topics ranging from economics $[44,45]$ over ecology [45] to coding theory [46] have been investigated using finite-time thermodynamics concepts.
} 


\section{Examples}

In this section, we present two examples for the optimization of chemical syntheses that proceed via nucleation and growth, e.g. from a melt. In the first example, the quantity to be maximized is the amount of desired product (only one solid modification exists). In the second example, two different solid modifications can form, and the quantity to be optimized is the difference in the amounts of the two products.

In both cases, we attempt to reduce the very complicated syntheses to their most elementary features. This allows us to construct highly simplified but at the same time quite generic models for the time evolution of the chemical system, such that the solutions of the optimal control problem can be derived analytically. While this simplification reduces the amount of realistic details of the model description, we can understand how the optimal control and the system interact, and furthermore we can analyze the influence of the parameters of the model on the behaviour of the optimal solution. Although the resulting solutions of the optimization problem usually are not very accurate in a quantitative sense, they typically represent the qualitative aspects of the optimal control of the real system correctly and can be used as guidance both in fine-tuning experiments and in the numerical solution of the optimal control problem when using much more realistic models of the synthesis process.

\subsection{Optimal control of a generic synthesis based on nucleation and growth}

The generic synthesis we are going to optimize is the production of the solid (crystalline) phase of a compound via cooling from the melt. However, the general 
analysis is also applicable to all syntheses that are based on nucleation-and-growth processes exhibiting the kind of temperature dependence described below. In contrast to e.g. a chemical reaction in the gas phase, two processes occur during the formation of a solid phase from the melt that exhibit very different dependences on the control parameters, here temperature: the nucleation of the desired phase and the subsequent growth of the nuclei. Their temperature dependence is opposite [50]: a reduction in temperature increases the rate of nucleation (down to the glass transition where the whole system freezes and no nuclei of critical size can be formed anymore), while an increase in temperature enhances diffusive processes and thus the growth of the nuclei and the product phase (up to the melting temperature, where the solid modification begins to fall apart again). ${ }^{2}$ Clearly, just choosing a single fixed temperature below the melting temperature and letting the system evolve for a time $\tau$ is very unlikely to yield the maximal amount of product within the finite time $\tau$.

\subsubsection{Mathematical model description}

In order to construct a model amenable to analytical analysis, the following simplifying assumptions are being made:

1. The amount of melt/volume $n_{\text {melt }}$ is assumed to greatly exceed the amount of solid material $n$ throughout the process. We lump all the material in the solid phase together in one variable $n(t)$, i.e. neither a cluster size distribution ${ }^{3}$ nor individual clusters are being considered in this averaged description. Since $n<<<n_{\text {melt }}=n_{\text {total }}-n \approx n_{\text {total }}$, the supercooled melt does not change due to transfer of material into the solid phase, and

\footnotetext{
${ }^{2}$ We consider only homogeneous nucleation, i.e. no externally induced heterogeneous nucleation takes place.

${ }^{3}$ Experience with modeling coarsening processes [51] has shown that many of the relevant features can be captured in averaged quantities such as the mean cluster size.
} 
we do not need to keep track of the amount of material in the supercooled melt explicitly but can treat it as an external constant that is implictly included in the parameters of the model.

2. The increase of the amount of desired product is governed by two processes, nucleation and growth. The growth rate is supposed to depend on the amount of material $n$ already present in the solid (crystalline) phase, in form of a power law $n^{r}$ $(0<r \leq 1),{ }^{4}$ while the nucleation rate is independent of $n$.

3. The temperature dependences of nucleation and growth follow power laws in a range between two reference temperatures $T_{1}<T<T_{0},\left(T_{0}-T\right)^{m}$ and $\left(T-T_{1}\right)^{l}$, respectively. No additional temperature dependence is assumed to be present. $T=T_{1}$ is the reference point (e.g. corresponding to the glass transition temperature), below which the nucleation rate rapidly drops from a maximum to zero because even local diffusion processes stop or become logarithmically slow and furthermore the thermodynamic barriers against the formation of critical nuclei rapidly increase. Similarly, below $T=T_{1}$, no significant growth can take place due to the slowness of diffusion processes at low temperatures. Without loss of generality, we can set $T_{1}=0$ for mathematical convenience. $T=T_{0}$ is the reference temperature (e.g. the melting point) above which no nucleation can take place. We also assume that above $T_{0}$ no growth can take place either. Thus the permitted temperature interval for the control is $T \in\left[0, T_{0}\right]$. Taking assumptions 2 and 3 together, the formula for the growth rate is given by $\frac{d n}{d t}=f(n, T)=A\left(T_{0}-T\right)^{m}+B T^{l} n^{r},(5)$

\footnotetext{
${ }^{4}$ If only one cluster were present, i.e. all the material were really accumulated in one single cluster, the most natural choice of $r$ would be $r=2 / 3$ reflecting the dependence of the growth rate on the size of the cluster surface. However, in reality, the total surface is the sum of the surface of many individual clusters, and this quantity is larger than the surface of a single big cluster, suggesting a larger value of $r$.
} 
with $m, l \geq 1$ and $0<r \leq 1$.

4. We assume that the internal temperature $T(t)$ of the system can nearly instantaneously adjust to changes in the control parameter, the external temperature $T_{e x t}(t)$, i.e. we can set

$T(t)=T_{e x t}(t)(6)$

in the formulation of the problem and treat $T(t)$ as the control.

5. Only homogeneous nucleation takes place. Also, we ignore effects due to preferred surfaces of the crystal for the growth processes. Furthermore, we ignore effects like the local depletion of the liquid phase (melt) or local changes of temperature due to the formation of the solid phase - the system is assumed to react quickly enough to adjust both the temperature and the local density of the liquid to the externally prescribed values.

The next step is the definition of the state variables of the system: In our simplified description, there are only two variables, $T$ and $n$. The external control variable is the applied temperature $T_{\text {ext }}$, which we can set equal to the internal temperature $T$. The quantity to be optimized is the total amount of (crystalline) solid phase $J=n(\tau)(7)$

On the other hand, there exist some empirical growth laws that are better represented by smaller values of $r$. 
generated in the interval $[0, \tau]$, where we assume that initially only melt was present $(n(0)=0)$. This means that we must maximize the integral of the growth rate of the solid phase $f(n, T)$,

$$
J=\int_{0}^{T} f(n(t), T(t)) d t,(8)
$$

with respect to the function $T(t)$. In addition, we need to add the constraint

$$
c(d n / d t, n, T)=\frac{d n}{d t}-f(n, T)=0(9)
$$

that describes the time evolution of $n$. This is included by augmenting the integral (c.f. supplementary material),

$$
J \rightarrow J_{\text {aug }}=\int_{0} f(n(t), T(t))+p(t)(d n / d t-f(n(t), T(t))) d t .(10)
$$

Thus we can formulate the optimal control problem as follows: Maximize the amount of product at the end of the duration of the synthesis, $n(\tau)=J$, by adjusting the temperature along the path, where the temperature is restricted to the interval $T \in\left[0, T_{0}\right]$, the time evolution of $n$ is given by eq. (5), and the initial amount of product $n(0)$ equals zero.

3.1.2 Solution of the optimal control problem 
The variation of $J_{\text {aug }}$ yields

$\delta J_{\text {aug }}=\int_{0}\left[\delta p(d n / d t-f)+\delta T\left(\frac{\partial f}{\partial T}-p \frac{\partial f}{\partial T}\right)+\delta n\left(\frac{\partial f}{\partial n}-p \frac{\partial f}{\partial n}-d p / d t\right)\right] d t,(11)$

where we have taken care of the variation of $d n / d t$ by performing an integration by parts, $p \delta(d n / d t)=p d \delta n / d t^{\prime \prime}="-(d p / d t) \delta n+$ vanishing surface terms. This yields for the optimization the following set of equations:

$\frac{d n}{d t}=f(n, T),(12 \mathrm{a})$

$(1-p(t)) \frac{\partial f}{\partial T}=0,(12 b)$

and

$\frac{d p}{d t}=(1-p(t)) \frac{\partial f}{\partial n} \cdot(12 \mathrm{c})$

Clearly, a trivial solution of eqs. (12b) and (12c) would be $p(t) \equiv 1$ for the whole time interval; however, in this case, there would be no constraint at all on the system's time evolution since then $\delta p(d n / d t-f(n, T))=0$ for all trajectories $n(t)$. Thus, we find that a non-trivial solution leads to

$\frac{\partial f}{\partial T}=-A m\left(T_{0}-T\right)^{m-1}+B l T^{l-1} n^{r}=0$ 
Note, however, that since $T$ is restricted to the interval $\left[0, T_{0}\right]$, the term associated with the variation of the control $T(t)$ can vanish either by fulfilling equation (13), or by $T(t)$ being piecewise constant on the boundary,

$T(t)=T_{0}$ or $T(t)=0,(14)$

such that $\delta T=0$ along these time intervals, with one or more switches between these values (a so-called bang-bang-type solution, c.f. supplementary material). Solving eq. (13) yields

$n=\left(\frac{m A}{l B} \frac{\left(T_{0}-T^{*}\right)^{m-1}}{T^{* l-1}}\right)^{(1 / r)}$

In order to decide, whether $T^{*}$ constitutes a local minimum or maximum of $f$, we next compute the second derivative

$\frac{\partial^{2} f}{\partial T^{2}}=m(m-1) A\left(T_{0}-T\right)^{m-2}+l(l-1) B T^{l-2} n^{r}(16)$

and insert the value of $n$ from eq. (15), i.e. we set $T=T^{*}$. We find $\partial^{2} f /\left.\partial T^{2}\right|_{T^{*}}>0$, and thus, $T^{*}(n)$ is a minimum for every value of $\mathrm{n}$. In particular, we find for the initial value $T^{*}(t=0)=T^{*}(n=0)=T_{0}$, and thus no production of the solid phase would take 
place. This result of solving the necessary conditions of the optimal control problem has produced the global minimum and not the maximum of the objective $n(\tau) .^{5}$

As a consequence, the optimal solution must be a bang-bang-type of solution, where $T$ switches between $T=0$ and $T=T_{0}$, and $d n / d t$ between the two boundary maxima of $f(n, T)$,

$f(T=0, n)=A T_{0}{ }^{m}$ and $f\left(T=T_{0}, n\right)=B T_{0}{ }^{l} n^{r},(17)$

respectively, at the value of

$n=n_{s}=\left(\frac{A}{B} T_{0}^{m-l}\right)^{\left(\frac{1}{r}\right)}$

where $f(T=0, n)=f\left(T=T_{0}, n\right)$.

For $T=0$,

$\frac{d n}{d t}=A T_{0}^{m},(19)$

and thus

$n(t)=n^{(1)}(t)=n\left(t_{0}\right)+A T_{0}^{m}\left(t-t_{0}\right) .(20)$

${ }^{5}$ Since $f \geq 0$ for all permitted values of $T$ and $n$, and $n(0)=0, n(\tau) \geq 0$ for every choice of the control $T(t)$. Thus, the solution found is obviously a global minimum. 
Similarly, for $T=T_{0}$,

$\frac{d n}{d t}=B T_{0}^{l} n^{r},(21)$

and

$n(t)=n^{(2)}(t)=\left((1-r) B T_{0}^{l}\left(t-t_{0}\right)+\left(n\left(t_{0}\right)\right)^{1-r}\right)^{(1 / 1-r)}$.

Note that for the initial condition $n\left(t_{0}\right)=0$, the differential equation for $n^{(2)}(t)$ is singular for $r<1$, and the appropriate solution is

$n^{(2)}(t)=0(23 \mathrm{a})$

instead of

$n^{(2)}(t)=\left((1-r) B T_{0}^{l}\left(t-t_{0}\right)\right)^{(1 / 1-r)} \cdot(23 \mathrm{~b})$

However, for any other value of $n\left(t_{0}\right)$, arbitrary close to 0 , eq. (23b) applies. A special case is $r=1$, where we find ${ }^{6}$

$n^{(2)}(t)=n\left(t_{0}\right) \exp \left(B T_{0}^{l}\left(t-t_{0}\right)\right) \cdot(24)$

\footnotetext{
${ }^{6}$ Note that eq. (24) is the limiting case of eq. (22) for $r=1$.
} 
Clearly, the initial choice of the temperature should be $T=0$, else we are just wasting valuable time. Since there should be only one switch to $T=T_{0}$, we can compute the optimal switching time

$t_{s} *=\left(\frac{B T_{0}^{l}}{\left(A T_{0}^{m}\right)^{1-r}}\right)^{(-1 / r)}$

by equating $n^{(1)}\left(t=t_{s}\right)=n_{s}$. From this follows the optimal trajectory for the amount of product

$$
n *(t)=\left\{\begin{array}{cr}
A T_{0}^{m} t, & 0 \leq t \leq t_{s}^{*} \\
\left((1-r) B T_{0}^{l}\left(t-t_{s}^{*}\right)+\left(A T_{0}^{m} t_{s}^{*}\right)^{1-r}\right)^{(1 / 1-r)}, t_{s}^{*}<t \leq \tau
\end{array} .\right.
$$

Table 1 shows $t_{s}^{*}, n_{s}=n^{*}\left(t=t_{s}^{*}\right)$, and the final amount of product $n *(t=\tau)$ for some useful choices of $l, m$ and $r$.

We note that after having realized that only one switch takes place from $T=0$ to $T=T_{0}$ at a time $t_{s}$, we could have first computed the general form of $n(t)$ parametrized by $t_{s}$,

$$
\hat{n}\left(t, t_{s}\right)=\left\{\begin{array}{c}
A T_{0}^{m} t, \\
\left((1-r) B T_{0}^{l}\left(t-t_{s}\right)+\left(A T_{0}^{m} t_{s}\right)^{1-r}\right)^{(1 / 1-r)}, t>t_{s}
\end{array},(27)\right.
$$

and then derived $t_{s} *$ by maximizing the function

$$
\tilde{n}\left(t_{s}\right)=\hat{n}\left(t=\tau, t_{s}\right)=\left((1-r) B T_{0}^{l}\left(\tau-t_{s}\right)+\left(A T_{0}^{m} t_{s}\right)^{1-r}\right)^{(1 / 1-r)}
$$


with respect to $t_{s}$. From this we can then compute the value of $n *(t)$ at the switching time $n *\left(t=t_{s}\right)=\hat{n}\left(t=t_{s}^{*}, t_{s}=t_{s}^{*}\right)$. In general, the derivative of $\hat{n}\left(t, t_{s}\right)$ with respect to $t$ is not defined at $t=t_{s}$; only for $t_{s}=t_{s} *$ the left- and right-derivative agree at $t=t_{s}$, since we now have $(d n / d t)^{(1)}\left(t=t_{s}\right)=(d n / d t)^{(2)}\left(t=t_{s}\right)$. For $t<t_{s}^{*}$, $(d n / d t)^{(2)}(t)<(d n / d t)^{(1)}(t)$, and for $t>t_{s}^{*},(d n / d t)^{(2)}(t)>(d n / d t)^{(1)}(t)$, i.e. the optimal choice of switching time ensures that the growth rate $f$ is maximized throughout the process for every value of $n$, a fact we have already indirectly exploited when choosing the bang-bang-type solution as the optimal one. ${ }^{7}$ In this context, we also note that exponential temperature dependences of the nucleation and/or growth rate would have led to the same kind of bang-bang-solution for the optimal control, as long as these exponential laws had exhibited the same monotonic decrease and increase, respectively, as the power laws for nucleation and growth we had assumed above. Furthermore, if $T_{1} \neq 0$, then we just have to replace $T_{0}$ by $T_{0}-T_{1}$ in all the formulas.

3.2 Optimal control of a generic nucleation-and-growth synthesis from the melt with competition between two different modifications

An interesting variation of the previous problem is the following one: Given a system, where two different modifications 1 and 2 are known to exist side-by-side in the solid state, how can one select the optimal temperature control such that modification 1 will be the preferred product of the synthesis via solidification from the melt? For

\footnotetext{
${ }^{7}$ The fact that $f \geq 0$ for all values of $\mathrm{n}$ and $\mathrm{n}(\mathrm{t})$ grows monotonically would allow us to solve the optimal control problem given by eq. (8) in an alternative fashion using Tsirlin's "averaged optimal control"
} 
concreteness, we will define as the quantity that is to be maximized the difference in the amounts of the two products $n_{1}(t)$ and $n_{2}(t)$

$\Delta(t)=n_{1}(t)-n_{2}(t)(29)$

after a finite time $\tau, \Delta(\tau)=n_{1}(\tau)-n_{2}(\tau)$. If both nucleation and growth rates of modification 1 are larger than the ones of modification 2, a decent heuristic might consist in just maximizing $n_{1}(\tau)$ and ignoring $n_{2}(\tau)$, thus reducing the task to the previously solved problem. But even in this special case, the optimal solution is more efficient, and we are clearly in trouble if modification 2 either nucleates or grows more quickly than modification 1.

3.2.1 Formulation of the optimal control problem

We will employ the same approximations as in the previous example. In particular, we assume that the general dependence of the nucleation and growth rates is the same for the two polymorphs, i.e., $m, l \geq 1$ and $r(0<r \leq 1)$ are the same for the two modifications. Thus, the difference between the two modifications resides only in the different rate parameters $A_{i}$ and $B_{i}, i=1,2$. Again, we assume that the total amount of starting material/volume, the (supercooled) melt, is essentially infinite compared to $n_{1}(t)$ and $n_{2}(t)$. A more subtle assumption is that the total time $\tau$, although potentially very large, should be smaller than the typical time scales where coarsening would take place and the thermodynamically stable modification would devour the unstable one. Taking these two assumptions together, we can assume that the two polymorphs 
nucleate and grow independently such that the amount of product of each modification never decreases during the process,

$\frac{d n_{i}}{d t}=f_{i}\left(n_{i}, T\right)=A_{i}\left(T_{0}-T\right)^{m}+B_{i} T^{l} n_{i}^{r} \geq 0(i=1,2)(30)$

for all permitted values of $T \in\left[0, T_{0}\right]$ and $n_{i} \geq 0$.

The state variables of the problem are the amounts of the two solid phases $n_{1}$ and $n_{2}$, and the temperature $T$ of the system that at the same time serves as the control variable. Thus we can formulate the optimal control problem as follows: Maximize the difference of the two products $\Delta=n_{1}-n_{2}$ at the end of the duration of the synthesis,

$$
\Delta(\tau)=J=\int_{0}^{1} f_{1}\left(n_{1}(t), T(t)\right)-f_{2}\left(n_{2}(t), T(t)\right) d t,(31)
$$

by adjusting the temperature along the path, where the temperature is restricted to the interval $T \in\left[0, T_{0}\right]$, the time evolution of $n_{i}$ is given by eq. (30), and the initial amount of product $n_{i}(0)$ equals zero $(i=1,2)$.

The two constraints $d n_{i} / d t=f_{i}\left(n_{i}, T\right)$ can again be included via the two Lagrangemultiplier functions $p_{i}(t)$ in an augmented functional

$$
J_{\text {aug }}=\int_{0}^{[}\left[\begin{array}{l}
\left(f_{1}\left(n_{1}(t), T(t)\right)-f_{2}\left(n_{2}(t), T(t)\right)\right)+p_{1}(t)\left(d n_{1} / d t-f_{1}\left(n_{1}(t), T(t)\right)\right)+ \\
+p_{2}(t)\left(d n_{2} / d t-f_{2}\left(n_{2}(t), T(t)\right)\right)
\end{array}\right] d t .
$$

we should chose $T$ such that $f$ is maximized. 
3.2.2. Solution of the optimal control problem

The variation of $J_{\text {aug }}$ proceeds analogously to the previous example, and yields the following set of equations:

$\frac{d n_{i}}{d t}=f_{i}\left(n_{i}, T\right) ;(i=1,2),(33 \mathrm{a})$
$\frac{d p_{i}}{d t}=\left(1-p_{i}\right) \frac{\partial f_{i}}{\partial n_{i}} ;(i=1,2),(33 \mathrm{~b})$

and $\left(1-p_{1}(t)\right) \frac{\partial_{1}}{\partial T}=\left(1-p_{2}(t)\right) \frac{\partial_{2}}{\partial T} \cdot(33 \mathrm{c})$

We note that the presence of two independent Lagrange-multiplier functions in eq. (33c) prevents us from applying the simple approach of the previous example, where the optimal solution could be directly identified by maximizing the function $f(T, n)$ for every value of $n$ with respect to $T$. Thus, we need to analyze the function

$\frac{d \Delta}{d t}\left(n_{1}, n_{2}, T\right)=f_{1}\left(n_{1}, T\right)-f_{2}\left(n_{2}, T\right),(34)$

whose integral is to be maximized with the constraints $d n_{i} / d t=f_{i}\left(n_{i}, T\right)$, in detail. In particular, we are interested in whether the optimal control $T^{*}(t)$ is an interior point in function space (in this case, we need to solve the full boundary value problem of the five coupled differential equations (33)) or whether we again are dealing with a bangbang-type solution. 
The analysis proves to be rather lengthy, and thus we just outline the procedure. We consider a set of points $n_{i}\left(t_{0}\right)=\breve{n}_{i}>0$ at some time $t_{0}>0$ that belong to the hypothetical optimal solution for $\Delta^{*}(t)=n_{1} *(t)-n_{2} *(t)$. What is now the temperature for which a maximal/minimal growth rate $(d \Delta / d t)\left(h_{1}, h_{2}, T\right)$ is found? Computing $\partial(d \Delta / d t) / \partial T$ and $\partial^{2}(d \Delta / d t) / \partial T^{2}$, we find that three cases can occur: 1. no interior extremum exists, i.e. we obviously have to choose one of the boundary values to increase $\Delta(t)$. 2. The interior extremum is a local minimum, and thus again $T^{*}=0$ or $T^{*}=T_{0} .3$. The interior extremum is a local maximum. But in this case, we find that $\Delta\left(t_{0}\right)$ is negative and both the nucleation and the growth rate of $n_{2}$ are larger than the ones for $n_{1}$. In this situation, the global optimal solution would have been to keep $T=T_{0}$ for the whole time interval $[0, \tau]$, i.e. we should not have attempted a synthesis in the first place since $n_{2}(\tau)>n_{1}(\tau)$ for all other choices of $T(t)$. Thus, the global solution to the optimal control problem that maximizes $\Delta(\tau)=n_{1}(\tau)-n_{2}(\tau)$ consists in a bang-bang-type solution where only one switch from $T=0$ to $T=T_{0}$ occurs. $^{8}$

The general solution to the optimal control problem is thus given by:

$$
\begin{aligned}
& T^{*}(t)=\left\{\begin{array}{c}
0,0 \leq t \leq t_{s}^{*} \\
T_{0}, t_{s}^{*}<t \leq \tau
\end{array},(35 \mathrm{a})\right. \\
& n_{i}^{*}(t)=\left\{\begin{array}{c}
A_{i} T_{0}^{m} t, \\
\left((1-r) B_{i} T_{0}^{l}\left(t-t_{s}^{*}\right)+\left(A_{i} T_{0}^{m} t_{s}^{*}\right)^{1-r}\right)^{(1 / 1-r)}, \begin{array}{l}
0 \leq t \leq t_{s}^{*} * \\
t_{s}^{*}<t \leq \tau
\end{array} ;(i=1,2) .
\end{array}\right.
\end{aligned}
$$

\footnotetext{
${ }^{8}$ Note that $\Delta^{*}(t)$ does not necessarily grow monotonically for the whole time interval (see supplementary material for more details).
} 
We still have to identify $t_{s} *$. To do so, we investigate $\hat{n}_{i}\left(t, t_{s}\right)$ and $\tilde{n}_{i}\left(t_{s}\right)$, and determine the value of $t_{s}$ that maximizes

$\tilde{\Delta}\left(t_{s}\right)=\tilde{n}_{1}\left(t_{s}\right)-\tilde{n}_{2}\left(t_{s}\right) \cdot(36)$

Several issues complicate the solution: For one, $t_{s} *$ can usually only be determined numerically by solving the equation

$$
\begin{aligned}
& \frac{d \tilde{n}_{1}\left(t_{s}\right)}{d t_{s}}=\frac{d \tilde{n}_{2}\left(t_{s}\right)}{d t_{s}} \Leftrightarrow \\
& \left.\left((1-r) B_{1} T_{0}^{l}\left(\tau-t_{s}\right)+\left(A_{1} T_{0}^{m} t_{s}\right)^{1-r}\right)^{(r / 1-r}\right)\left(-B_{1} T_{0}^{l}+\left(A_{1} T_{0}^{m}\right)^{1-r} t_{s}^{-r}\right)=. \\
& \left.=\left((1-r) B_{2} T_{0}^{l}\left(\tau-t_{s}\right)+\left(A_{2} T_{0}^{m} t_{s}\right)^{1-r}\right)^{(r / 1-r}\right)\left(-B_{2} T_{0}^{l}+\left(A_{2} T_{0}^{m}\right)^{1-r} t_{s}^{-r}\right)
\end{aligned}
$$

This equation can have 0,1 or 2 solutions $t_{s} *$ in the interval $[0, \tau]$, and furthermore $\tilde{\Delta}\left(t_{s}^{*}\right)$ have to be compared with the value of $\tilde{\Delta}\left(t_{s}\right)$ at the boundary of the time interval, $\tilde{\Delta}\left(t_{s}=0\right)$ and $\tilde{\Delta}\left(t_{s}=\tau\right)$. A further subtle complication arises from the singularity of the differential equation (21): we need to distinguish between $t_{s}^{*}=0$ (which implies that $T=T_{0}$ for the whole time and no solid is produced) and $t_{s}^{*}=\varepsilon(1>>\varepsilon>0)$ (which implies that we have tiny initial nuclei of modifications 1 and 2 that grow for the whole time interval). Finally, the type and number of solutions $t_{s} *$ depends on the relative size of $A_{1}, A_{2}, B_{1}$ and $B_{2}$ characterizing the two nucleation and growth rates in general. Thus, eight different cases must be studied individually: $B_{1}=B_{2}, A_{1}>A_{2}(1)$; $B_{1}=B_{2}, A_{1}<A_{2}(2) ; B_{1}>B_{2}, A_{1}=A_{2}(3) ; B_{1}<B_{2}, A_{1}=A_{2}(4) ; B_{1}>B_{2}, A_{1}>A_{2}$ (up to 3 subcases) (5); $B_{1}>B_{2}, A_{1}<A_{2}$ (up to 6 subcases) (6); $B_{1}<B_{2}, A_{1}>A_{2}$ (up to 6 subcases) (7), $B_{1}<B_{2}, A_{1}<A_{2}$ (8). The subcases reflect the values of these rates, and their rates of 
change, at $t=0$ and $t=\tau$, and also the total time $\tau$ available. Some of the general types of solutions one finds are: 1$) t_{s}^{*} \approx t_{s}^{(1)} *$ (essentially maximize the amount of modification 1; especially if $B_{1}>B_{2}$ and $\tau$ large), 2) $t_{s}^{*}=0$ (perform no synthesis at all), 3) $t_{s}^{*} \approx \tau$ (essentially permit only nucleation, especially if $B_{1} \leq B_{2}, A_{1}>A_{2}$ ), 4) $t_{s}^{*}=\varepsilon$ (after an extremely short nucleation phase switch to the growth phase, especially if $B_{1}>B_{2}, A_{1}<A_{2}$ ). Note that we only have one switch in temperature along the optimal trajectory: If $B_{1}>B_{2}$, then we will stay in the growth phase once it became advantageous to switch to higher temperature, and if $B_{1}<B_{2}$, then we time the switch to the growth phase (if we switch at all!) in such a fashion that a possible switch back to low temperature would occur precisely once the total allotted time has been used up (and thus no further switch is needed).

Tables 2 and 3 summarize the results for $m=l=1, r=1$ and $m=l=1,0<r<1$, respectively. Since interior values of $t_{s}^{*}$ must be computed numerically, in the general case we can only give their location relative to the optimal switching times $t_{s}^{(1)} *$ and $t_{s}^{(2)} *$ we would obtain if we wanted to maximize the amount of modification 1 and 2 , respectively, regardless of the amount of the competing phase (regarding the formulas for $t_{s}^{(1)} *$ and $t_{s}^{(2)} *$, c.f. eq. (25)). In the supplementary material, we discuss the simplest case, $m=l=1, r=1$, in some more detail to illustrate the procedure, and similarly address the case $m=l \neq 1, r \neq 1$. Note that the rich solution structure shown in the tables can make it difficult to design simple heuristic guidelines for achieving the objective: even relatively small changes in the various parameters in eq. (30) or the total synthesis time can lead to a switch to a different regime of optimal solutions. 
Note that we can use the same basic approach to address the more general case, where $m_{1} \neq m_{2}, l_{1} \neq l_{2}$, and/or $r_{1} \neq r_{2}$. We find the same kind of bang-bang-type solutions, but the number of cases to be analyzed grows enormously, and even a tabular overview is not very helpful. Thus we are not presenting the detailed results for this general case; for specific systems with given experimental values of $m_{i}, l_{i}$ and $r_{i}$, the most efficient way would be to treat the optimal control problem analogously to the case $m_{1}=m_{2}, l_{1}=l_{2}$ and $r_{1}=r_{2}$ analyzed above, and then numerically solve the analogue to eq. (37) for the switching time.

\section{Discussion}

4.1. Summary of results and application to a real system (glycerol)

In the previous section, we have formulated two optimal control problems for a nucleation-and-growth based synthesis using a simplified but nevertheless quite general model for the nucleation and growth rates of the product phase as function of temperature and product already present. We have shown that these problems, the maximization of the yield of one solid phase and the maximization of the difference between the amounts of two solid phases, can be solved and that we obtain solutions of the bang-bang-type, with a switch from $T=T_{1}\left(\approx T_{\text {glass }}\right)$ to $T=T_{0}\left(\approx T_{\text {melt }}\right)$, in both cases. To make these problems analytically accessible, many simplifying assumptions were required. But the fact that we were able to reach a quite general solution that holds for very generic laws describing the temperature dependence of nucleation and growth of 
the nuclei, yields much insight into the general optimal control problem of this type of syntheses and the qualitative aspects of its solution.

A natural question is to what extent this ,phenomenological“ optimal control result can be applied in a quantitative fashion: Can we plug numbers based on experiments into the solutions in tables $1-3$ and e.g. find $t_{s}^{*}$ anywhere near realistic values?

Obviously, the first step would be a fit of the model parameters, $A_{i}, B_{i}, T_{0}, T_{1}, m, l, r$, to experimental data describing nucleation and growth rates as function of temperature. Next we would calculate the appropriate switching time, either by inserting the parameters into the formulas in table 1 or by numerically solving eq. (37).

As a specific example, we consider the application of the optimal control formalism to the synthesis of glycerol crystals. Here, the goal is to maximize the yield of the crystalline phase within the finite time $\tau$. From the literature [50], we find that the nucleation rate in the supercooled melt increases very rapidly upon cooling to a maximum at $T_{1} \approx-65^{\circ} \mathrm{C}$ and then rather abruptly drops to zero even before the glass transition temperature $T_{\text {glass }} \approx-85^{\circ} \mathrm{C}$ is reached [53]. The growth rate of the crystallites increases quickly with increasing temperature to a maximum at about $T_{0} \approx 0^{\circ} \mathrm{C}$, and then rapidly decreases towards the melting temperature $T_{\text {melt }} \approx+18^{\circ} \mathrm{C}$ [54]. Fitting power laws to the data yields for the model parameters: $A \approx 2.4 \times 10^{-13} / \mathrm{sec} \mathrm{cm}^{3} \mathrm{~K}^{9}$, $B \approx 0.3 / \sec c m K^{3}, T_{0}-T_{1} \approx 65 K, m \approx 9, l \approx 3$, and $r \approx 2 / 3$. If we plug these values into the formulas in table 1 , we find a switching time of $t_{s}^{*} \approx 2 \mathrm{sec}$. Thus, the optimal 
solution consists of keeping the melt for about 2 seconds at about $-65^{\circ} C$, and then let the nuclei generated grow at the maximum rate at a temperature of about $0^{\circ} C .{ }^{9}$

\subsection{Analysis of the approximations}

As mentioned above, several approximations and simplifications had been necessary, in order to make the optimal control problem analytically accessible. Perhaps the most drastic one is the description of the product phase by a single variable $n(t)$, ignoring the fact that we are actually dealing with a distribution of clusters of various sizes.

However, in the past it has proven to be quite successful to focus on the time evolution of an ,average“ cluster while modeling processes involving nucleation of clusters instead of trying to follow the full distribution, e.g. in various models of primary crystallization [55], coarsening [51] or in the optimization of the gas-liquid transition [13]. Thus only two variables would be needed to describe the solid phase, the total amount of crystalline material $n(t)$ and the number of clusters generated, $N_{C}(t)$. Since the $N_{C}$ clusters are treated as identical, they obey the same growth law. As a consequence, the fact that $n(t)$ represents not only one cluster but several ones can be included in a heuristic fashion by choosing the parameter $r$ in the model (see eq. (5)) to be different from $r=2 / 3$. In particular, we note from the glycerol example that less than 10 nuclei $/ \mathrm{cm}^{3}$ have been generated by the time $t_{s} *$ is reached, and thus treating the system as if all the solid material were combined into one effective crystallite should be quite reasonable. Having to modify $r$ is only a small price to pay for eliminating

\footnotetext{
${ }^{9}$ There is some uncertainty about the size of the critical nuclei. We assumed a critical cluster size of 1000 atoms when determining the parameter A. If a critical cluster contains only 100 atoms (a lower bound), A would be smaller by about a factor 10 , and $t_{s} *$ would be reduced by a factor of about 3 .
} 
$N_{C}(t)$ as an independent variable. The situation is slightly different in the case of two competing modifications where $t_{s}^{*}=\tau$ can be the optimal solution, and thus the exponent $r$ would vary as a function of $n(t)$ since $N_{C}(t)$ ranges from one to many nuclei. However, in this case the optimal solution is independent of the value of $r$ (the growth phase of the nuclei is actually never reached), and thus the model again describes the features of the growth process relevant for the optimal control problem.

The second approximation concerns the use of power laws to model the nucleation and growth rates. Of course, these rates do not drop infinitely fast to zero for $\left(T_{\text {glass }}<\right) T<T_{1}$ and $\left(T_{\text {melt }}>\right) T>T_{0}$, respectively. However, we note that for $T<T_{1}$, the growth rate remains zero, and analogously the nucleation rate is zero for $T>T_{0}$. From the point of view of optimizing the amount of final product, there is clearly nothing to be gained from ever selecting a temperature below $T_{1}$ or above $T_{0}$, and thus we can restrict the range of feasible values of the control, i.e. the temperature, to the interval $\left[T_{1}, T_{0}\right]$. In particular, we note that as long as the rates are monotonic in the interval $\left[T_{1}, T_{0}\right]$, the general solution will be of the bang-bang-type, and one usually can find some reasonable power-law approximation for the temperature dependence of the rates within the relevant interval.

The situation is more subtle if the two competing modifications should exhibit different values for $T_{1}^{(i)}$ and $T_{0}^{(i)}$, e.g. $T_{1}^{(1)}<T_{1}^{(2)}<T_{0}^{(1)}<T_{0}^{(2)}$. While the models as such are still an appropriate description of the rates of each of the two polymorphs within $\left[T_{1}^{(i)}, T_{0}^{(i)}\right]$, it is not clear, whether we still have a bang-bang-type of solution of the optimal control problem. In principle, we need to consider the large interval $\left[T_{1}^{(1)}, T_{0}^{(2)}\right]$ as the range of 
feasible values of the control parameter, but then the peaks in the nucleation rate for modification 2 and in the growth rate for modification 1 do not occur at the boundaries. But if we choose the small interval $\left[T_{1}^{(2)}, T_{0}^{(1)}\right]$, it can happen that at $T_{1}^{(2)}$ and $T_{0}^{(1)}$ the growth rate of modification 1 or the nucleation rate for modification 2, respectively, are still substantially different from zero while on the other hand the nucleation rate of modification 1 at $T_{1}^{(2)}$ and the growth rate of modification 2 at $T_{0}^{(1)}$ are still far from their maximum values. Clearly, for neither of the two intervals we would expect a straigthtforward bang-bang solution where the boundaries of the interval could serve as the optimal temperature values. Thus, a full re-analysis of the optimal control problem is called for if $T_{0}^{(1)} \neq T_{0}^{(2)}$ or $T_{1}^{(1)} \neq T_{1}^{(2)}$. In contrast, slightly different power laws of the temperature dependence of the nucleation and growth rates, $l_{1} \neq l_{2}$ and/or $m_{1} \neq m_{2}$, do not pose big problems, and even different values of $r_{1} \neq r_{2}$ will only complicate the equations (e.g. lead to additional solutions in the analogue to eq. (37) for $r_{1} \neq r_{2}$ ). But these changes would not preclude an analytical analysis, as long as $T_{0}^{(1)}=T_{0}^{(2)}$ and $T_{1}^{(1)}=T_{1}^{(2)}$, because we can still expect that a bang-bang-type solution is the optimal one.

Another important approximation is the range of allowed synthesis times $\tau$. Clearly, if $\tau \rightarrow \infty$, the amount of melt $n_{\text {melt }}$ will decrease and $n(t)$ will become comparable unless we keep adding supercooled melt to the system effectively keeping the density of solid material small compared to $n_{\text {melt }}$ inside the synthesis chamber. Similarly, for large times, coarsening of the cluster size distribution will take place. However, the neglect of coarsening effects is not critical in the context of our model because the average cluster size still keeps increasing, and thus the lumped approximation that only considers the total amount of solid phase $n(t)$ encompasses and averages out possible coarsening 
effects, with the consequence that coarsening does not have much of an influence on the optimal control problem. Regarding the decrease of $n_{\text {melt }}$, we note that if we are maximizing the amount of a single solid phase, the transition to a pure growth stage takes place rather early independent of the total synthesis time. Thus, the decrease in $n_{\text {melt }}$ does not affect the optimal control problem as such, and it will only be noticeable in the fact that $n(t)$ reaches a limiting value corresponding to the intrinsic density of the solid phase itself (instead of growing to infinity as the formulas in section 3.1 suggest).

Again, additional complications arise for two competing modifications. Here, cases can occur (c.f. tables 2 and 3), where the switching time equals $\tau$ or is relatively close to $\tau$. These situations only occur if we need to stay for a very long time in the nucleation stage that favors modification 1 , in order to balance the faster growth of the nuclei belonging to modification 2. This fact will not change much if the amount of melt is being depleted, and it will still be favorable to stay at $T=T_{1}$ for nearly the whole time if we want to maximize $n_{1}-n_{2}$. Much more critically, coarsening processes can be of great importance when two modifications compete, since for sufficiently large synthesis times and/or sufficiently unstable nuclei/crystallites, the thermodynamically stable modification will eliminate the metastable solid phase even in finite time. If such effects are to be taken into account, one would need to construct a considerably more sophisticated model of the nucleation-and-growth synthesis with competing modifications (possibly including an approximate cluster size distribution). It is very likely that such a model can only be solved numerically.

Finally, we have assumed that the system reacts instantaneously both to changes in the applied external temperature and to local changes in temperature and melt-density 
caused by the nucleation of clusters of critical size or by the attachment of atoms from the melt to the crystallites. Taking these effects into account will lead to optimal control solutions that are approximately of the bang-bang-type, as long as the relaxation times are much smaller than $t_{s} *$. This can be expected to hold for so-called microreactors, e.g. in the increasingly popular "lab-on-a-chip". If the relaxation times are rather large, however, a full re-analysis of the optimal control problem is required taking the flow properties and the thermal conductivity of the melt into account, and the more complicated set of differential equations one would derive using the variational approach would most likely have to be solved numerically.

\subsection{Outlook}

The discussion of the various approximations in the previous subsection implicitly suggests a number of future projects in the optimal control of nucleation-and-growth based syntheses. Most straightforward is the analysis of the optimal control problem with competing polymorphs for $l_{1} \neq l_{2}, m_{1} \neq m_{2}$ and/or $r_{1} \neq r_{2}$. Similarly, one could replace the power laws in eqs. (5) and (30) by more general functions involving exponentials such as $\exp \left[-\Delta G(T) / k_{B} T\right]$ that are often used to describe activation barriers to the nucleation of clusters of critical size [50]. As we mentioned earlier, the general type of solution is not going to change as long as these functions are monotonically increasing and decreasing with temperature, respectively, although the case-by-case analysis is going to be much more complicated and numerical solutions will be unavoidable. 
Next, one would want to address the case where the maxima of growth and nucleation rates of different modifications do not occur at the same temperature. A more substantial extension of the model would be the inclusion of coarsening processes between the competing polymorphs, where one probably will need to add at least the number of clusters or equivalently the average size of the clusters for each modification to the state variables describing the system. Furthermore, one would add conversion terms between the two modifications as function of cluster size in the rate equations for $\left(d n_{i} / d t\right)(t)$ and $\left(d N_{C} / d t\right)^{(i)}(t)$.

A further issue that deserves to be studied is the competition between homogeneous and heterogeneous nucleation. As a first step, one might want to study purely heterogeneous nucleation-and-growth on the same level of complexity as was done in this work for homogeneous nucleation, followed by a combination of both nucleation processes. Another level of sophistication of the models involves the homogeneity of the spatial distribution of the solid clusters within the supercooled melt. In contrast to the gas phase where a well-stirred reactor approximation is quite realistic, the condensed phases are rather slow in relaxing to (thermodynamic) equilibrium after e.g. the external temperature has been changed or latent heat has been released during a nucleation event. Modeling these delays is clearly important but also quite challenging, in particular with regard to the solution of the associated optimal control problems.

Clearly, many more possible extensions of the models employed here can be proposed and investigated. However, another very interesting question is to what extent the optimal control problems we have studied can be applied to other nucleation-andgrowth based syntheses besides the growth of a solid phase from a melt. In particular, it 
would be worthwhile to investigate the growth of crystals from solution, or the crystallization inside an amorphous matrix e.g. during the synthesis of metastable crystalline modifications via the low-temperature atom deposition method [56,57].

Finally, one might want to consider the place of our two optimal control problems in the framework of finite-time thermodynamics. The generic laws and guidelines incorporated in eq. (3), and in eqs. (9) and (10) in the supplementary material, are based on the assumption that we are moving between two equilibrium states within a finite time that is still large enough for the system to stay close to the equilibrium path it would follow in the infinite-time limit. Such considerations would be applicable e.g. when one tries to refine our simple model by taking the delays in the relaxation to local equilibrium into account. However, the generic aspect of the type of problems we consider in this work is that they are „open-ended“, i.e. we want to produce as much of a solid crystalline phase as possible within the given time, and not minimize the amount of work needed to transform a certain amount of melt into solid in finite time while staying close to the melting (i.e. equilibrium) temperature. In particular, we note that both for loss of availability and entropy dissipation, the Hessian of the energy and the entropy, respectively, can serve as a positive definite metric and this allows the definition of a proper thermodynamic distance (c.f. eq. (4)). This is not the case for the processes studied in this work, where the analogous quantity, the second derivative of the amount of product with respect to the temperature, $\partial^{2} n / \partial T^{2}$, is not necesssarily positive or negative definite for the whole process and thus no thermodynamic length can be computed.

The optimal control problems we consider are more similar to the maximization of the product of a chemical reaction in the gas phase studied in earlier work [6,7]. For the 
optimization of the reactions $n A \leftrightarrow m B$ [6] it was found that the distance between the optimal and the equilibrium curves, $T *\left(N_{\text {product }}\right)$ and $T_{e q}\left(N_{\text {product }}\right)$, respectively, in $\left(N_{\text {product }}, T\right)$-space was approximately constant. In contrast to those finite-time thermodynamics problems where entropy production or loss of availability were minimized, this distance did not go to zero for $\tau \rightarrow \infty$, however. But while in the gas phase reaction there existed a well-defined equilibrium curve for the amount of product as function of temperature to serve as a reference, an analogous quantity is more difficult to discern in a nucleation-and-growth synthesis, since three (metastable) „equilibrium states“ of the system have to be taken into account, each of which might serve as a reference point: the (supercooled) melt, the (macroscopic) crystal and the clusters (of critical or larger size). Clearly, if one could establish that such a constancy in $\Delta T$ also holds in optimally controlled nucleation-and-growth processes, this would allow us to employ this information in determining an optimal path for those systems that do not easily yield to the optimal control methods described in section 3 and the supplementary material.

To a certain degree, a comparison can be drawn with two consecutive chemical reactions $A \leftrightarrow B \leftrightarrow C$, where the melt, the critical nuclei and the crystalline solid would correspond to the chemical species "A", "B" and "C", respectively. Such a system has been studied [7] using Tisrlin's averaged optimal control [52] to maximize the intermediary species "B". In contrast to this study, the model presented in section 3.1 assumes that no back-reactions take place, and due to the bang-bang-type of solution nucleation and growth processes do not take place in appreciable amounts at the same time. Furthermore, unless one were to specify individual large crystals instead of the total amount of crystalline phase as the objective, the species "B" and "C", i.e. the 
critical nuclei and the larger crystals, both contribute to the objective of the optimal control $n(\tau)$. Thus, the analogy does not hold in all details. As a consequence, we never encounter a "maximal useful time" that is less than the total time available. Due to the non-reversibility of the processes incorporated in the model and the fact that in the optimal solution either nucleation or growth takes place, the same holds true even if we were to maximize only the number of critical nuclei since obviously the maximum would be achieved by remaining in the nucleation phase for the whole duration $\tau$. But in general, it should be possible to pursue this analogy further, especially once one includes the number of clusters $N_{C}$ as an independent state variable and modifies the objective to be the amount of crystalline phase that is present in the form of large crystals.

Similarly, one might try to compare the second optimization problem with the case of two competing chemical reactions, $A \leftrightarrow B$ and $A \leftrightarrow C$. Again, no back-reactions are included in the melt-to-crystalline material system, in contrast to a standard e.g. gas phase reaction, where both a possible transformation $C \leftrightarrow B$ (via $C \leftrightarrow A \leftrightarrow B$ ) and the existence of equilibrium concentrations for "A", "B", and "C" at a given temperature are implied. Furthermore, eq. (30) describes a rather unusual pair of reaction rates, since each is a sum of two terms, one of which increases (monotonically) with the amount of product present, and thus represents a positive feedback built into the reaction, in contrast to the usual slowing down of the net reaction rate due to the increase of the back-reaction rate with increasing amount of product. Thus, while such a comparison would be valid in principle, this unusual reaction rate and general set-up makes it difficult to apply one's intuition based on competing gas phase reactions. ${ }^{10}$

\footnotetext{
${ }^{10}$ This problem of competing gas phase reactions does not appear to have been treated within the context of finite-time thermodynamics so far.
} 
The rather mathematical study presented here might appear to be far removed from the everyday cares and worries of the experimental synthetic chemist. But even in the nonindustrial context greater efficiency is something to be strived for; e.g., achieving large differences in the amount of the two competing phases might greatly simplify the subsequent purification stage. And the purposeful synthesis of only one of several different metastable modifications is one of the great challenges of experimental solid state chemistry.[19,20] It is hoped that the results obtained in this investigation will provide a starting point for further analyses of the optimal control of nucleation-andgrowth based syntheses, where the construction of more detailed models and their solution by theory needs to be complemented by robust, quantitative measurement data that only experiment can supply.

Bibliography

[1] S. Sienutycz, Optimization in Process Engineering, WNT, Warsaw 1991.

[2] K. G. Denbigh, Chem. Eng. Sci. 1958, 8, 125.

[3] R. Aris, Z. Elektrochem. 1961, 65, 229.

[4] F. Horn, U. Troltenier, Chemie-Ing.-Tech. 1960, 32, 382.

[5] B. Mansson, B. Andresen, I\&EC Process Design \& Development 1986, 25, 59.

[6] J. C. Schön, B. Andresen, J. Phys. Chem. 1996, 100, 8843.

[7] T. A. Bak, P. Salamon, B. Andresen, J. Phys. Chem. A 2002, 106, 10961.

[8] O. C. Mullins, R. S. Berry, J. Phys. Chem. 1984, 88, 723. 
[9] J. C. Schön, B. Andresen, Ind. Eng. Chem. Res. 1996, 35, 2327.

[10] E. S. Jimenez, P. Salamon, R. Rivero, C. Rendon, K. H. Hoffmann, M. Schaller, B. Andresen, Ind. Eng. Chem. Res. 2004, 43, 7566.

[11] C. A. O. Nascimento, R. Giudici, R. Guardani, Comp. \& Chem. Eng. 2000, 24, 2303.

[12] A. Bejan, Heat and Fluid Flow 1987, 8, 258.

[13] M. Santoro, J. C. Schön, M. Jansen, Phys. Rev. E 2007, 76, 061120.

[14] L. Mutihac, R. Mutihac, Analyt. Chim. Acta 2008, 612, 1.

[15] J. F. MacGregor, T. Kourti, Control Eng. Pract. 1995, 3, 403.

[16] S. M. Senkan, Nature 1998, 394, 350.

[17] M. A. R. Meier, R. Hoogenboom, U. S. Schubert, Macromol. Rapid Comm. 2004, $25,21$.

[18] J. C. Schön, M. Jansen, Angew. Chem. Int. Ed. 1996, 35, 4025.

[19] M. Jansen, Angew. Chem. Int. Ed. 2002, 41, 3746.

[20] M. Jansen, in: Turning Points in Solid-State, Materials and Surface Science, Eds.

K. M. Harris and P. P. Edwards, RSC Publ., Cambridge 2008, p. 22.

[21] J. C. Schön, M. Jansen, Int. J. Mater. Res. 2009, 100, 135.

[22] D. E. Kirk, Optimal Control Theory, Prentice Hall, Englewood 1970.

[23] S. Sienutycz, P. Salamon, Eds., Finite-Time Thermodynamics and

Thermoeconomics, Taylor\&Francis, New York 1990.

[24] B. Andresen, R. S. Berry, A. Nitzan, P. Salamon, Phys. Rev. A 1977, 15, 2086.

[25] M. H. Rubin, Phys. Rev. A 1979, 19, 1272\&1279.

[26] R. A. Giaggoli, Thermodynamics: Second Law Analysis, Amer. Chem. Soc.

Washington, D.C. 1980.

[27] R. C. Tolman, P. C. Fine, Rev. Mod. Phys. 1948, $20,51$. 
[28] F. L. Curzon, B. Ahlborn, Amer. J. Phys. 1975, 43, 22.

[29] P. Salamon, A. Nitzan, B. Andresen, R. S. Berry, Phys. Rev. A 1980, 21, 2115.

[30] J. M. Gordon, Amer. J. Phys. 1991, 59, 551.

[31] K. H. Hoffmann, S. J. Watowich, R. S. Berry, J. Appl. Phys. 1985, 58, 2125.

[32] G. R. Brown, S. Snow, B. Andresen, P.Salamon, Phys. Rev. A 1986, 34, 4370.

[33] J. M. Gordon, I. Rubinstein, Y. J. Zarmi, J. Appl. Phys. 1990, 67, 81.

[34] R. K. Pathria, P. Salamon, J. D. Nulton, J. Phys. A 1998, 31, 3171.

[35] A. Kodal, B. Sahin, T. Yilmaz, Energy Convers. Managem. 2000, 41, 607.

[36] S. Wu, J. Chen, Appl. Energy 2005, 80, 349.

[37] A. Bejan, Entropy Generation through Heat and Fluid Flow, Wiley-Interscience, New York 1982.

[38] J. M. Gordon, Solar Energy 1988, 40, 457.

[39] F. Wu, L. Chen, F. Sun, C. Wu, G. Guo, Q. Li, Open Syst. Inform. Dyn. 2006, 13, 55.

[40] B. Lin, J. Chen, Phys. Scripta 2008, 77, 055005.

[41] A. Durmayaz, O. S. Sogut, B. Sahin, H. Yavuz, Prog. Energy Comb. Sci. 2004, 30, 175.

[42] V. N. Orlov, R. S. Berry, Phys. Rev. A 1990, 42, 7230.

[43] J. C. Schön, J. Chem. Phys. 1996, 105, 10072.

[44] P. Salamon, J. Komlos, B. Andresen, J. D. Nulton, Math. Soc. Sci. 1987, 13, 153.

[45] G. Lebon, D. Jou, J. Casas-Vazquez, Finite-Time Thermodynamics: Economy,

Ecology, and Heat Engines, Springer, Berlin 2008.

[46] J. D. Flick, P. Salamon, B. Andresen, Inform. Sci. 1987, 42, 239.

[47] P. Salamon, R. S. Berry, Phys. Rev. Lett. 1983, 51, 1127.

[48] F. Weinhold, J. Chem. Phys. 1975, 63, 2479\&2484\&2488. 
[49] P. Salamon, J. D. Nulton, E. Ihrig, J. Chem. Phys. 1984, 80, 436.

[50] I. Gutzow, J. Schmelzer, The Vitreous State: Thermodynamics, Structure, Rheology, and Crystallization, Springer, Berlin 1995.

[51] I. M. Lifshitz, V. V. Slyozov, J. Phys. Chem. Solids 1961, 19, 35.

[52] L. Rozonoer, A. M. Tsirlin, Avtom. Telemekh. 1983, 1, 70 (Autom. Remote Control (Engl. Transl.) 1983, 44, 55).

[53] A. A. Chernov, Formation of Crystals, in: Modern Crystallography, vol. 3, Nakua Publishers, Moscow 1980.

[54] M. Volmer, M. Marder, Z. Phys. Chem. A 1931, 154, 97.

[55] P. Bruna, D. Crespo, R. Gonzalez-Cinca, E. Pineda, J. Appl. Phys. 2006, 100, 054907.

[56] D. Fischer, M. Jansen, J. Amer. Chem. Soc. 2002, 124, 3488.

[57] D. Fischer, M. Jansen, Angew. Chem. Int. Ed. 2002, 41, 643. 


\section{Tables:}

Table 1: $t_{s}^{*}, n^{*}\left(t_{s}^{*}\right), n^{*}(\tau)$ for the maximization of the one-component system, for different choices of $m, l$ and $r$.

\begin{tabular}{|c|c|c|c|}
\hline$m=l=1, r=1$ & $t_{s}^{*}$ & $n^{*}\left(t_{s}^{*}\right)$ & $n^{*}(\tau)$ \\
\hline$m=l=1,0<r<1$ & $1 / B T_{0}$ & $A / B$ & $(A / B) \exp \left[B T_{0} \tau-1\right]$ \\
\hline$m, l \geq 1, r=1$ & $A^{(1-r / r} / T_{0} B^{(1 / r)}$ & $(A / B)^{(1 / r)}$ & {$\left[(1-r) B T_{0} \tau+r(A / B)^{(1-r / r)}\right]^{(1 / 1-r)}$} \\
\hline$m, l \geq 1,0<r<1$ & $1 / B T_{0}^{l}$ & $(A / B))^{m-l}$ & $(A / B))_{0}^{m-l} \exp \left[B T_{0}^{l} \tau-1\right]$ \\
\hline & $\left(A^{(1-r / r} / B^{(1 / r)}\right) T_{0}^{(m-l / r-m)}$ & $\left.[(A / B))_{0}^{m-l}\right]^{(1 / r)}$ & {$\left[(1-r) B T_{0}^{l} \tau+r\left(A / B_{0}^{T_{0}^{m-l}}\right)^{(1-r / r}\right)^{(1 / 1-r)}$} \\
\hline
\end{tabular}


Table 2: Case-by-case list of the approximate location of $t_{s}^{*}, \tilde{\Delta}^{\prime}(0), \tilde{\Delta}^{\prime}(\tau)$, and $\Delta^{*}(\tau \rightarrow \infty)$, where applicable, for the maximization of the difference between the two modifications, $\Delta(\tau)=n_{1}(\tau)-n_{2}(\tau)$, for $m=l=1, r=1$. The results for $m, l>1$ can be found by replacing $A_{i} T_{0}$ and $B_{i} T_{0}$ by $A_{i} T_{0}^{m}$ and $B_{i} T_{0}^{l}$, respectively, both in the conditions defining the various cases and subcases, and in the actual results. Subcases (i), (ii) and (iii) are defined in the supplementary material. Note that subcases (i) and (ii) are only applicable for short values of $\tau$.

\begin{tabular}{|c|c|c|c|c|c|}
\hline & $\tilde{\Delta}^{\prime}(0)$ & $\tilde{\Delta}^{\prime}(\tau)$ & $t_{s}^{*}$ & $\Delta^{*}(\tau \rightarrow \infty)$ & Comment \\
\hline$B_{1}=B_{2}, A_{1}>A_{2}$ & $>0$ & $<0$ & $1 / B T_{0}$ & $\Delta^{*}(\tau) \rightarrow \frac{A_{1}-A_{2}}{B_{1}} \exp \left[B_{1} T_{0} \tau-1\right]$ & \\
\hline$B_{1}=B_{2}, A_{1}<A_{2}$ & $<0$ & $>0$ & 0 & 0 & No synthes. \\
\hline$B_{1}>B_{2}, A_{1}=A_{2}$ & $>0$ & $<0$ & $0<t_{s}^{*}<t_{s}^{(1)} *$ & $\Delta^{*}(\tau) \rightarrow A_{1} T_{0} t_{s} * \exp \left[B_{1} T_{0}\left(\tau-t_{s}^{*}\right)\right]$ & \\
\hline$B_{1}<B_{2}, A_{1}=A_{2}$ & $<0$ & $>0$ & $0 ; \tau$ & 0 & $\begin{array}{l}\text { No synthes. } \\
\text { / only nucl. }\end{array}$ \\
\hline$B_{1}>B_{2}, A_{1}>A_{2}$ & $>0$ & $<0$ & $0<t_{s}^{*}<t_{s}^{(1)} *<t_{s}^{(2)} *$ & $\Delta^{*}(\tau) \rightarrow A_{1} T_{0} t_{s} * \exp \left[B_{1} T_{0}\left(\tau-t_{s}^{*}\right)\right]$ & \\
\hline $\begin{array}{l}B_{1}>B_{2}, A_{1}<A_{2} \\
\text { i) (see suppl.) }\end{array}$ & $<0$ & $>0$ & 0 & $\tau \rightarrow \infty$ not applicable; $\Delta^{*}(\tau)=0$ & $\begin{array}{l}\text { No synthes. } \\
\text { (increase } \tau \\
\text { to reach case } \\
\text { iii) }\end{array}$ \\
\hline $\begin{array}{l}B_{1}>B_{2}, A_{1}<A_{2} \\
\text { ii) (see suppl.) }\end{array}$ & $>0$ & $>0$ & $t_{s}^{*}=t_{s 1} *<t_{s}^{(1)} *<t_{s}^{(2)} *$ & $\tau \rightarrow \infty$ not applicable; $\Delta^{*}(\tau)>0$ & \\
\hline $\begin{array}{l}B_{1}>B_{2}, A_{1}<A_{2} \\
\text { iii) (see suppl.) }\end{array}$ & $>0$ & $<0$ & $0<t_{s} *<t_{s}^{(1)} *<t_{s}^{(2)} *$ & $\Delta^{*}(\tau) \rightarrow A_{1} T_{0} t_{s} * \exp \left[B_{1} T_{0}\left(\tau-t_{s}^{*}\right)\right]$ & \\
\hline $\begin{array}{l}B_{1}<B_{2}, A_{1}>A_{2} \\
\text { i) (see suppl.) }\end{array}$ & $>0$ & $<0$ & $t_{s}^{(2)} *<t_{s}^{(1)} *<t_{s}^{*}<\tau$ & $\begin{array}{c}\tau \rightarrow \infty \text { not applicable; } \\
\Delta *(\tau)>\left(A_{1}-A_{2}\right) T_{0} \tau>0\end{array}$ & \\
\hline $\begin{array}{l}B_{1}<B_{2}, A_{1}>A_{2} \\
\text { ii) (see suppl.) }\end{array}$ & $<0$ & $<0$ & $t_{s}^{(2)} *<t_{s}^{(1)} *<t_{s}^{*}=t_{s 2} *$ & $\begin{array}{c}\tau \rightarrow \infty \text { not applicable; } \\
\Delta *(\tau)=\tilde{\Delta}\left(t_{s 2}\right)>\left(A_{1}-A_{2}\right) T_{0} \tau>0\end{array}$ & \\
\hline $\begin{array}{l}B_{1}<B_{2}, A_{1}>A_{2} \\
\text { iii) (see suppl.) }\end{array}$ & $<0$ & $>0$ & $\tau$ & $\left(A_{1}-A_{2}\right) T_{0} \tau>0$ & Only nucl. \\
\hline
\end{tabular}




\begin{tabular}{|c|c|c|c|c|c|}
\hline$B_{1}<B_{2}, A_{1}<A_{2}$ & $<0$ & $>0$ & 0 & 0 & No synthes. \\
\hline
\end{tabular}


Table 3: Case-by-case list of the approximate location of $t_{s}^{*}, \tilde{\Delta}^{\prime}(0), \tilde{\Delta}^{\prime}(\tau)$, and $\Delta^{*}(\tau \rightarrow \infty)$, where applicable, for the maximization of the difference between the two modifications, $\Delta(\tau)=n_{1}(\tau)-n_{2}(\tau)$, for $m=l=1,0<r<1$. The results for $m, l>1$ can be found by replacing $A_{i} T_{0}$ and $B_{i} T_{0}$ by $A_{i} T_{0}{ }^{m}$ and $B_{i} T_{0}^{l}$, respectively, both in the conditions defining the various case and subcases, and in the actual results. Subcases (i), (ii) and (iii) and their subcases are defined in the supplementary material. Note 1: A priori, it is not possible to decide, whether the global maximum occurs at the boundary $\left(t_{s}^{*}=\varepsilon\right)$ or at the interior maximum. Note 2: A priori, it is not possible to decide, whether the global maximum occurs at the boundary $\left(t_{s}^{*}=\tau\right)$ or at the interior maximum (if an interior maximum exists at all).

\begin{tabular}{|c|c|c|c|c|c|}
\hline & $\tilde{\Delta}^{\prime}(0)$ & $\tilde{\Delta}^{\prime}(\tau)$ & $t_{s}^{*}$ & $\Delta^{*}(\tau \rightarrow \infty)$ & Comment \\
\hline$B_{1}=B_{2}, A_{1}>A_{2}$ & $>0$ & $<0$ & $t_{s}^{(2)} *<t_{s}^{(1)} *<t_{s} *$ & $\left.\left((1-r) B_{1} T_{0} \tau\right)^{(r / 1-r}\right)\left[\left(A_{1} T_{0} t_{s}^{*}\right)^{1-r}-\left(A_{2} T_{0} t_{s}^{*}\right)^{1-r}\right] /(1-r)$ & \\
\hline$B_{1}=B_{2}, A_{1}<A_{2}$ & $<0$ & $>0$ & 0 & 0 & No synthes. \\
\hline$B_{1}<B_{2}, A_{1}=A_{2}$ & $<0$ & $>0$ & $0, \tau$ & 0 & $\begin{array}{c}\text { No synthes. / } \\
\text { only nucl. }\end{array}$ \\
\hline $\begin{array}{l}B_{1}>B_{2}, A_{1}>A_{2} \\
\text { i) (see suppl.) }\end{array}$ & $>0$ & $<0$ & $t_{s}^{(2)} *<t_{s}^{(1)} *<t_{s} *$ & $>\left(B_{1}^{1 /(1-r)}-B_{2}^{1 /(1-r)}\right)\left[(1-r) T_{0} \tau\right]^{1 /(1-r)}$ & \\
\hline $\begin{array}{l}B_{1}>B_{2}, A_{1}>A_{2} \\
\text { iii) (see suppl.) }\end{array}$ & $>0$ & $<0$ & $t_{s}^{*}=t_{s}^{(1)} *=t_{s}^{(2)} *$ & $>\left(B_{1}^{1 /(1-r)}-B_{2}^{1 /(1-r)}\right)\left[(1-r) T_{0} \tau\right]^{1 /(1-r)}$ & \\
\hline $\begin{array}{l}B_{1}>B_{2}, A_{1}<A_{2} \\
\text { i) (see suppl.) }\end{array}$ & $<0$ & $>0$ & $\varepsilon$ & $\left(B_{1}^{1 /(1-r)}-B_{2}^{1 /(1-r)}\right)\left[(1-r) T_{0} \tau\right]^{1 /(1-r)}$ & $\begin{array}{c}\text { Infinitesimal } \\
\text { nucl. + growth }\end{array}$ \\
\hline $\begin{array}{l}B_{1}>B_{2}, A_{1}<A_{2} \\
\text { ii) (see suppl.) }\end{array}$ & $<0$ & $>0$ & $\varepsilon$ & $\left(B_{1}^{1 /(1-r)}-B_{2}^{1 /(1-r)}\right)\left[(1-r) T_{0} \tau\right]^{1 /(1-r)}$ & $\begin{array}{l}\text { Infinitesimal } \\
\text { nucl. + growth }\end{array}$ \\
\hline
\end{tabular}




\begin{tabular}{|c|c|c|c|c|c|}
\hline $\begin{array}{c}B_{1}>B_{2}, A_{1}<A_{2} \\
\text { iii)c) (see suppl.) }\end{array}$ & $>0$ & $>0$ & $t_{s}^{*}=t_{s 1} *<t_{s}^{(1)} *<t_{s}^{(2)} *<t_{s 2} *$ & $>\left(B_{1}^{1 /(1-r)}-B_{2}^{1 /(1-r)}\right)\left[(1-r) T_{0} \tau\right]^{1 /(1-r)}$ & \\
\hline $\begin{array}{l}B_{1}<B_{2}, A_{1}>A_{2} \\
\text { ii) (see suppl.) }\end{array}$ & $>0$ & $<0$ & $t_{s}^{*}<t_{s}^{(1)} *<t_{s}^{(2)} *$ & $>\left(A_{1}-A_{2}\right) T_{0} \tau$ & \\
\hline $\begin{array}{c}B_{1}<B_{2}, A_{1}>A_{2} \\
\text { iii)b) (see suppl.) }\end{array}$ & $<0$ & $>0$ & $\tau$ & $\left(A_{1}-A_{2}\right) T_{0} \tau$ & Only nucl. \\
\hline $\begin{array}{c}B_{1}<B_{2}, A_{1}>A_{2} \\
\text { iii)c) (see suppl.) }\end{array}$ & $>0$ & $>0$ & $\begin{array}{c}t_{s}^{(2)} *<t_{s}^{(1)} *<t_{s 1} *=t_{s}^{*}<t_{s 2} * \\
\text { or } t_{s}^{*}=\tau\end{array}$ & $\geq\left(A_{1}-A_{2}\right) T_{0} \tau$ & Note 2 \\
\hline $\begin{array}{l}B_{1}<B_{2}, A_{1}>A_{2} \\
\text { iii)d) (see suppl.) }\end{array}$ & $<0$ & $<0$ & $t_{s}^{(1)} *<t_{s}^{(2)} *<t_{s 1} *<t_{s 2} *=t_{s} *$ & $>\left(A_{1}-A_{2}\right) T_{0} \tau$ & \\
\hline
\end{tabular}

